\title{
mRNA expression profile analysis reveals a C-MYC/miR-34a pathway involved in the apoptosis of diffuse large $B$-cell lymphoma cells induced by Yiqichutan treatment
}

\author{
LINZHU ZHAI $^{1 *}$, YUANYUAN ZHAO ${ }^{2 *}$, ZEYU LIU $^{1 *}, \mathrm{JIE} \mathrm{WU}^{3}$ and LIZHU LIN ${ }^{1}$ \\ ${ }^{1}$ Cancer Center, The First Affiliated Hospital of Guangzhou University of Chinese Medicine, Guangzhou, Guangdong 510405; \\ ${ }^{2}$ Department of Medical Oncology, Sun Yat-sen University Cancer Center; State Key Laboratory of Oncology in South China; \\ Collaborative Innovation Center for Cancer Medicine, Guangzhou, Guangdong 510060; ${ }^{3}$ Project Department, \\ Forevergen Biosciences Co., Ltd., Guangzhou, Guangdong 510000, P.R. China
}

Received June 19, 2019; Accepted March 17, 2020

DOI: $10.3892 /$ etm.2020.8940

\begin{abstract}
Diffuse large B-cell lymphoma (DLBCL) is the most common subtype of adult non-Hodgkin's lymphoma (NHL). While DLBCL is sensitive to chemotherapy, a certain percentage of patients with DLBCL experience relapse. Previous studies have indicated that Yiqichutan treatment, which was developed to treat NHL, can inhibit DLBCL cell growth, but the mechanism is not fully understood. The present study identified 991 differentially expressed mRNAs, with 498 upregulated and 493 downregulated $(\mathrm{P}<0.05)$, in SUDHL-6 cells exposed to Yiqichutan. The underlying pathways included the Jak/Stat and PI3K signaling pathways. In total, six representative mRNAs were selected for validation with reverse transcription-quantitative PCR (RT-qPCR), and a strong correlation was identified between the RT-qPCR results and microarray data. Since the transcription factor C-MYC is involved in both the Jak/Stat and PI3K signaling pathways, C-MYC and its associated microRNA (miR) were selected for further analysis. It was found that knockdown of C-MYC increased miR-34a expression levels, inhibited forkhead box P1 (Foxp1) expression levels and promoted DLBCL cell apoptosis. In addition, the miR-34a mimics further enhanced the role of C-MYC knockdown. It was demonstrated that, the expression levels of apoptotic factors Bax and poly (ADP-ribose) polymerase were significantly upregulated with C-MYC knockdown and miR-34a mimics in SUDHL-6 cells, while the $\mathrm{Bcl} 2$ expression level was significantly reduced. Moreover, Yiqichutan treatment increased miR-34a expression levels and
\end{abstract}

Correspondence to: Dr Lizhu Lin, Cancer Center, The First Affiliated Hospital of Guangzhou University of Chinese Medicine, 16 Jichang Road, Guangzhou, Guangdong 510405, P.R. China E-mail: lizhulin@hotmail.com

*Contributed equally

Key words: microRNA-34a, C-MYC, Yiqichutan, microarray induced apoptosis, as well as reducing Foxp1 expression level in SUDHL-6 cells. Therefore, the present results suggested that Yiqichutan treatment affected DLBCL cells via several signaling pathways. Furthermore, Yiqichutan may inhibit the proliferation of DLBCL cells by blocking the C-MYC/miR-34a signaling pathway.

\section{Introduction}

Diffuse large B-cell lymphoma (DLBCL) is highly invasive and is the most common subtype of adult non-Hodgkin's lymphoma (NHL), accounting for 30-40\% of all NHL (1). Rituximab combined with CHOP-based chemotherapy is considered a curative treatment, and $60-70 \%$ of patients can be treated following first-line immune-chemotherapy, while $30-40 \%$ of patients relapse at a certain point during the disease (2-4). Therefore, from the perspective of developing a comprehensive treatment, it is crucial to identify novel therapeutics for DLBCL. As important components of complementary and alternative medicines, Traditional Chinese Medicines (TCM) have long been practiced in China and are gaining popularity in the western countries, such as the USA, UK and Germany (5-7). According to TCM, factors impacting DLBCL development include deficiencies in the internal organs and the accumulation of phlegm (8). Previous studies have shown that Yiqichutan as a decoction, which was developed to treat NHL, can inhibit DLBCL cell growth and significantly reduce phosphorylated-AKT expression level in DLBCL cells $(8,9)$. However, the underlying mechanism of Yiqichutan for treating DLBCL remains elusive.

Expression profile analysis is widely used to identify genetic variations in oncological research (10), thus, the present study use microarray analysis to investigate the effects of Yiqichutan treatment on DLBCL mRNAs. Previous studies have shown that the interaction between microRNAs (miRNAs/miRs) and transcription factors may be associated with the progression from low-grade to a highly aggressive lymphoma, such as in DLBCL (11). Furthermore, previous studies have shown that miR-34a acts as a strong tumor suppressor in solid cancer types, including lung $(12,13)$, prostate (14), pancreatic (15), 
renal (16) and metastatic bone cancer (17). Moreover, epigenetic inactivation of miR-34a by aberrant expression levels can be found in $18 \%$ of NHLs (18). Bioinformatic target prediction combined with functional analyses has revealed that the oncogene C-MYC mRNA can be regulated by miR-34a (19), and acts via post-transcriptional control of the transcription factor forkhead box (Fox) protein family, especially Foxp1 which is a hematopoietic oncoprotein overexpressed in DLBCL $(20,21)$. Therefore, the C-MYC/miR-34a pathway may be closely related to the occurrence and development of DLBCL. Thus, the present study investigated the impact of Yiqichutan treatment on the regulation of the C-MYC/miR-34a signaling pathway. It was found that, Yiqichutan treatment affected DLBCL cells via several signaling pathways and that Yiqichutan may inhibit the proliferation of DLBCL cells by blocking the C-MYC/miR-34a signaling pathway.

\section{Materials and methods}

Cell culture. Human DLBCL SUDHL-6 cells were gifted from The Cancer Hospital of Sun Yat-sen University Cancer Center, and have been authenticated using short tandem repeat matching analysis. The cells were cultured in RPMI-1640 medium (Gibco; Thermo Fisher Scientific, Inc.) with 10\% FBS (Gibco; Thermo Fisher Scientific, Inc.) and $100 \mathrm{U} / \mathrm{ml}$ penicillin/streptomycin (Gibco; Thermo Fisher Scientific, Inc.) at $37^{\circ} \mathrm{C}$ with $5 \% \mathrm{CO}_{2}$, and saturated humidity. The medium was replaced every other day.

Yiqichutan. Yiqichutan treatment was composed of a decoction prepared with $15 \mathrm{~g}$ American ginseng, $15 \mathrm{~g}$ Pinellia ternata, $15 \mathrm{~g}$ Cremastra appendiculata, $30 \mathrm{~g}$ Ranunculus ternatus, 15 g Fritillaria thunbergii, 15 g Herba sarcandrae, $10 \mathrm{~g}$ fried batryticated silkworm and $30 \mathrm{~g}$ Ganoderma lucidum (http://www.theplantlist.org). The raw materials were purchased from The First Affiliated Hospital of Guangzhou University of Chinese Medicine, and cooled with purified water three times for $30 \mathrm{~min}$ each, at 10, 8 and 8 times the weight of the raw materials (w/v), sequentially. Pinellia ternate was boiled $\left(100^{\circ} \mathrm{C}\right)$ for $30 \mathrm{~min}$ prior to use. The boiled water was collected, filtered and condensed to $2 \mathrm{~g}$ of raw materials per $\mathrm{ml}$ in the final preparation, then sealed in bags with a sealing machine and stored at $4^{\circ} \mathrm{C}$ for subsequent use.

mRNA microarray expression profiling, Gene Ontology (GO) analysis and pathway enrichment analysis. Microarray hybridization was carried out by Shanghai Genechem Co., Ltd. for the present study. After treatment for $48 \mathrm{~h}\left(37^{\circ} \mathrm{C}\right)$ with $14.65 \mathrm{mg} / \mathrm{ml}$ Yiqichutan or a control (sterile water) for SUDHL-6 cells, three cell samples were collected from the Yiqichutan treatment group and the control group. For each treatment group of cells, the total RNA extracted with TRlzol $^{\circledR}$ (Invitrogen; Thermo Fisher Scientific, Inc.) was purified according to the manufacturer's instructions and reverse transcribed into cDNA using M-MLV reverse transcriptase (cat. no. M1705; Promega Corporation) according to the manufacturer's instructions. The purified, sense-strand cDNA was fragmented and labelled by terminal deoxynucleotidyl transferase using a GeneChip WT Pico kit (cat. no. 902623; Applied Biosystems; Thermo Fisher Scientific, Inc.). The purified labeled cDNA was then subjected to hybridization using Affymetrix Clariom S Genechip WT Pico reagent kit (Clariom S Assay; Affymetrix; Thermo Fisher Scientific, Inc.) following the manufacturer's instructions. An Affymetrix Gene ChIP scanner was used to scan the microarrays, and the CEL files generated were analyzed using Affymetrix Expression Console software (version 4.0; Affymetrix; Thermo Fisher Scientific, Inc.). The data were normalized using logarithmic transformation. The low expressed genes detected in all the samples were selected for elimination and further data analysis. Only genes with an ANOVA $\mathrm{P} \leq 0.05$ were considered as differentially expressed between the experimental and control groups. GO analysis provides a controlled vocabulary to describe gene and gene product attributes in any organism (http://www.geneontology.org). Fisher's exact test was used to detect overlap, which would be expected by chance, between the differentially expressed list and the GO annotation list. The P-value denotes the significance of GO term enrichment among differentially expressed genes $(\mathrm{P} \leq 0.05)$. The Kyoto Encyclopedia of Genes and Genomes (KEGG) is a set of high-throughput genes and protein pathways (22). KEGG analyses can be found in the following database: http://www.genome.jp/kegg. $\mathrm{P} \leq 0.05$ (EASE-score, Fisher P-value or hypergeometric P-value) denotes the significance of the pathway correlations.

Extraction of total RNA and reverse transcription-quantitative PCR (RT-qPCR). At $48 \mathrm{~h}$ after treating with Yiqichutan, $5-10 \times 10^{6}$ cells per group were collected and total RNA was isolated using TRIzol ${ }^{\circledR}$ reagent (cat. no. TR118-500; Invitrogen; Thermo Fisher Scientific, Inc.) according to the manufacturer's protocol. A total of $1 \mu \mathrm{g} 1$ extracted RNA was used to synthesize cDNA in a reaction using oligo (dT) primers and M-MLV reverse transcriptase (cat. no. M1705; Promega Corporation) according to the manufacturer's instructions. PCR amplification of mRNA and miRNA was performed with a GoTaq ${ }^{\circledR}$ qPCR Master mix (Promega Corporation; cat. no. A6002) on an RT-qPCR instrument (Bio-Rad Laboratories, Inc.; MiniOpticon). The following thermocycling conditions used were: Initial denaturation at $95^{\circ} \mathrm{C}$ for $120 \mathrm{sec}$; 40 cycles of $95^{\circ} \mathrm{C}$ for $15 \mathrm{sec}$, and a final extension at $60^{\circ} \mathrm{C}$ for $30 \mathrm{sec}$. The PCR products were calculated with

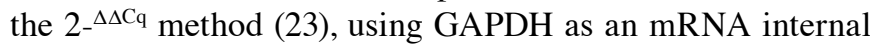
control and U6 as a miRNA internal control. The primers used for amplifying specific genes are shown in Table SI.

Cell transfection. A total of $50 \mathrm{nM}$ miRNA mimics (Shanghai GenePharma Co., Ltd) and $200 \mathrm{nM}$ small interfering RNAs (siRNAs; Shanghai GenePharma Co., Ltd.) were used for transfection. The siRNA targeting C-MYC sequences were as follows: C-MYC siRNA forward, 5'-GAACACACAACGUCU UGGATT-3' and reverse, 5'-UCCAAGACGUUGUGUGUU CTT-3'; and siC-MYC-2 forward, 5'-AACGUUAGCUUCAC CAACATT-3' and reverse, 5'-UGUUGGUGAAGCUAACGU UTT-3'. The hsa-miR-34a-5p mimics sequences were as follows: Forward, 5'-UGGCAGUGUCUUAGCUGGUUGU-3' and reverse, 5'-AACCAGCUAAGACACUGCCAUU-3'. Lipofectamine 2000 transfection reagent (Thermo Fisher Scientific, Inc.) was used for cell transfection, following the manufacturer's instructions. Cells were collected $48 \mathrm{~h}$ after 
A

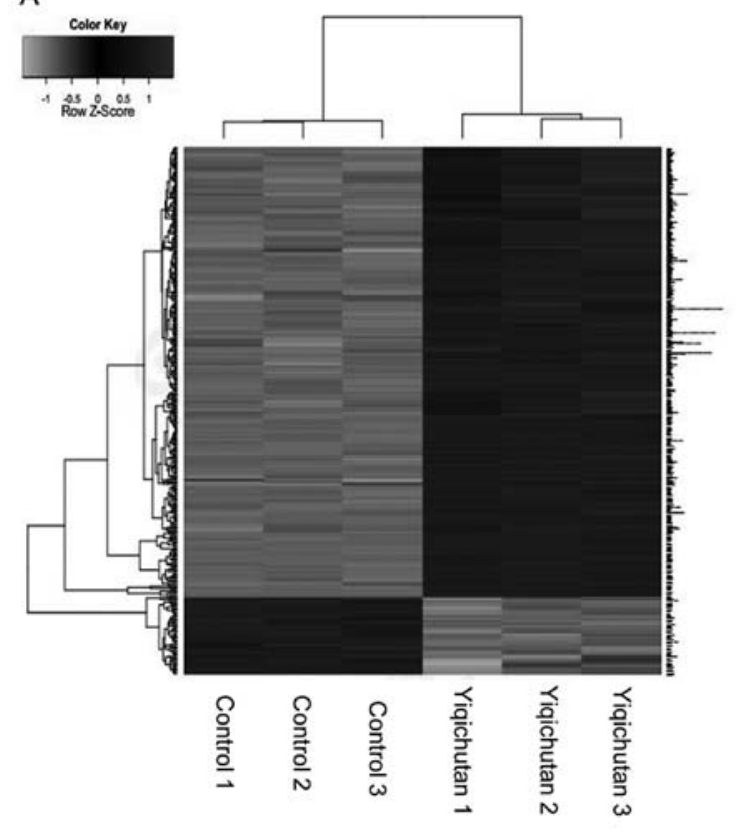

B

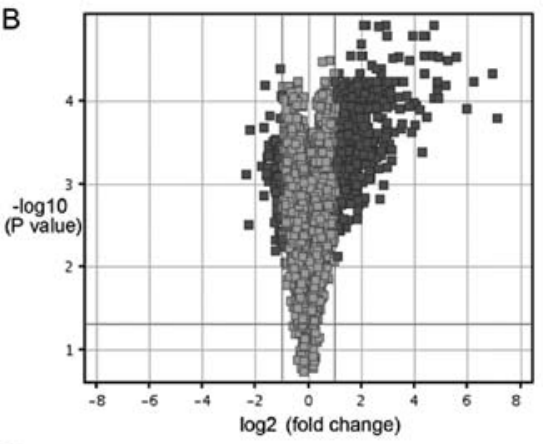

C

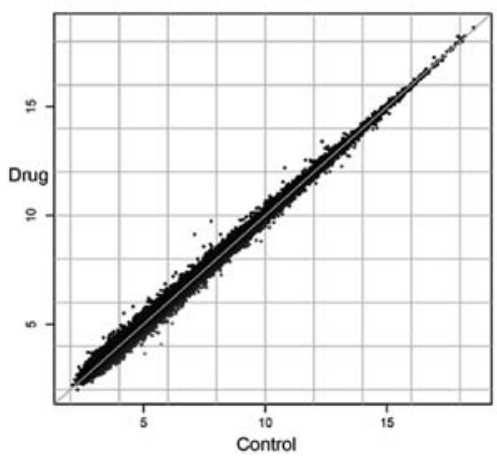

Figure 1. Identification of differentially expressed mRNAs in SUDHL-6 cells. (A) Heat map of altered genes exposed to Yiqichutan treatment. P<0.05. In total, three replicates of the Yiqichutan group and the control group were analyzed. (B) Volcano and (C) scatter plots showed significant variation of mRNA expression between the Yiqichutan and control groups.

transfection and the C-MYC expression levels were determined by RT-qPCR as described above.

Western blot analysis. After being treated and cultured, the cells were harvested and protein extractions were prepared with a modified RIPA buffer (Beyotime Institute of Biotechnology) with $0.5 \%$ SDS in the presence of a proteinase inhibitor cocktail (Beyotime Institute of Biotechnology). A bicinchoninic acid protein concentration kit (cat. no. P0011; Beyotime Institute of Biotechnology) was used to determine protein concentration. A total of $20 \mu \mathrm{g}$ protein/lane was separated by $6 \%$ or $12 \%$ SDS-PAGE. Proteins were then transferred onto a PVDF membrane. The membranes were then blocked with 5\% BSA containing TBS- $0.05 \%$ Tween- 20 for $\sim 2 \mathrm{~h}$ at room temperature and incubated overnight at $4^{\circ} \mathrm{C}$ with the following primary antibodies: Anti-C-MYC (1:1,000; cat. no. ab32072; Abcam), anti-Foxp1 (1:1,000; cat. no. 4402; Cell Signaling Technology, Inc.) and GAPDH (1:1,000; cat. no. ab8245; Abcam). After washing with PBS containing $0.1 \%$ Tween-20 three times for 5 min each, the membranes were incubated with horseradish peroxidase-linked immunoglobulin G (1:1,000; cat. no. 7074; Cell Signaling Technology, Inc.) secondary antibody for $2 \mathrm{~h}$ at room temperature. The membranes were developed using an enhanced chemiluminescence system (Guangzhou Forevergreen Biosciences Co., Ltd.).

MTS assay. The viability of SUDHL-6 cells was evaluated by cell proliferation rates measured with an MTS Cell Proliferation assay kit (Promega Corporation; cat. no. G3580) following the manufacturer's protocols. SUDHL-6 cells were incubated in $5 \% \mathrm{CO}_{2}$ and at $37^{\circ} \mathrm{C}$ in 96 -well microtiter plates at $\sim 80 \%$ confluency and treated with different concentrations of 4, 8, 12, 16 and $20 \mathrm{mg} / \mathrm{ml}$ of Yiqichutan. After 24, 48 and
$72 \mathrm{~h}$, SUDHL- 6 cells were incubated with $20 \mathrm{~g}$ of MTS reagent for $4 \mathrm{~h}$ at $37^{\circ} \mathrm{C}$ in a humidified culture chamber supplied with $5 \% \mathrm{CO}_{2}$. The optical density $490 \mathrm{~nm}$ values were measured using a plate reader (Diatek DR-200Bs), and were used to evaluate cell proliferation and viability. For statistical analysis, three replicates were carried out.

Cell apoptosis analysis. Flow cytometry was used to perform cell apoptosis analysis. The cells were incubated at $37^{\circ} \mathrm{C}$ in six-well plates and treated with $200 \mathrm{nM}$ siRNAs or $50 \mathrm{nM}$ miRNA mimics, or with different concentrations of Yiqichutan $(4,8,12,16$ and $20 \mathrm{mg} / \mathrm{ml})$ for $48 \mathrm{~h}$. Cells were collected by centrifugation at $800 \mathrm{xg}$ and $4^{\circ} \mathrm{C}$ for $5 \mathrm{~min}$ and washed with PBS buffer. The cells were stained with $5 \mu \mathrm{l}$ APC Annexin V (BD Biosciences) and $10 \mu \mathrm{l}$ 7-AAD (BD Biosciences) for $15 \mathrm{~min}$ in the dark at room temperature, then analyzed by flow cytometry (FACSAria III; BD Biosciences). A FACScalibur flow cytometer (BD Biosciences) was used for analysis.

Statistical analysis. Data are presented as the mean \pm SD. SPSS 18.0 statistical software (SPSS, Inc.) and GraphPad Prism 7.0 (GraphPad Software, Inc.) were used for analysis. A Student's t-test was performed to test the difference between two groups and one-way ANOVA tests with Tukey's test were used to analyze differences among groups. $\mathrm{P}<0.05$ was considered to indicate a statistically significant difference.

\section{Results}

Comparison of gene expression profiles between the two groups. The expression profiles of 21,448 common genes were found in all the samples, and a total of 991 differentially expressed genes were identified in SUDHL-6 cells treated with Yiqichutan. Of 
A

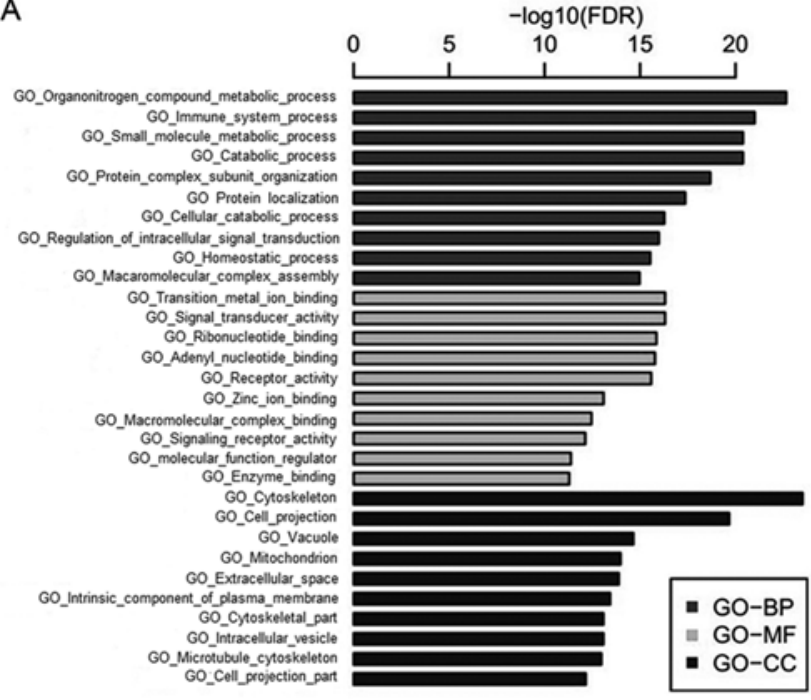

B

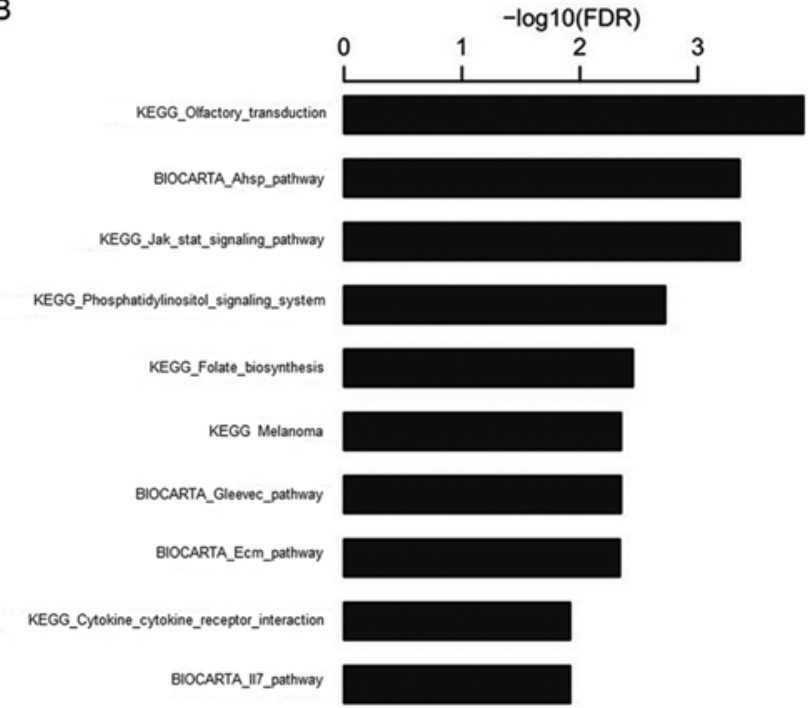

Figure 2. GO and KEGG pathway analysis. (A) GO enrichment analysis of differentially expressed genes. (B) KEGG pathway enrichment analysis of differentially expressed genes. BP, biological process; MF, molecular function; CC, cellular component; GO, Gene Ontology; KEGG, Kyoto Encyclopedia of Genes and Genomes.

these genes, 498 were upregulated and 493 genes were downregulated, and a hierarchical clustering of mRNA expressions is shown Fig. 1A. Furthermore, the volcano and scatter plots indicated a significant variation of mRNA expression level between the control group and the treatment group (Fig. 1B and C).

GO and KEGG pathway analysis. To investigate potential gene and gene product enrichments in molecular functions, biological processes and cellular components, $\mathrm{GO}$ analysis was performed with the differentially expressed mRNAs (Fig. 2A). The differentially expressed genes were subjected to pathway analysis based on the KEGG database and the associated 10 pathways are shown in Fig. 2B, which included the Jak/Stat and PI3K signaling pathways. Thus, the present results suggested that these pathways may contribute to the pathogenesis and biochemical characteristics of DLBCL, and that Yiqichutan may play a role in treating DLBCL via these pathways.

Confirmation of the microarray data by RT-qPCR analysis. While 991 mRNAs were found to have significant changes in SUDHL-6 cells after treating with Yiqichutan in microarray assays, to assess the reliability of the microarray data several significantly differentially expressed genes were selected for RT-qPCR analysis, including C-MYC, Mouse double minute 2 (MDM2), fibroblast growth factor 2 (FGF2), Dual specificity protein phosphatase 16 , suppressor of cytokine signaling 2 (SOCS2) and bone morphogenetic protein receptor type 2 . The RT-qPCR results were in line with the microarray data as both showed the same trends $(\mathrm{P}<0.05$; Fig. 3). The oncogene C-MYC encodes the nuclear transcription factor C-MYC, which is involved in both the Jak/Stat and PI3K signaling pathways (24), which were the molecular pathways identified in the KEGG analysis. Therefore, the C-MYC pathway was selected for further analysis.

Knockdown of C-MYC increases miR-34a expression level and targets Foxpl. In order to identify whether the transcription factor C-MYC was involved in the regulation of miR-34a to affect the progression of DLBCL, SUDHL- 6 cells were transfected with C-MYC siRNAs and miR-34a mimics. It was found that the expression levels of C-MYC and miR-34a in SUDHL-6 cells were significantly suppressed and increased after treatment with C-MYC and miR-34a mimic, respectively (Fig. 4A-C). Moreover, C-MYC inhibition may increase the expression level of miR-34a and reduce Foxp1 expression level. In addition, miR-34a overexpression and decreased Foxp1 expression levels were more significant after transfection with both C-MYC siRNAs and miR-34a mimics (Fig. 4D-F). Thus, the present results suggested that C-MYC may be a key upstream mediator, and Foxp1 is a key downstream mediator of miR-34a in SUDHL-6 cells. Therefore, the effect of C-MYC knockdown on cell proliferation and apoptosis were analyzed. The RT-qPCR results indicated that Bax and poly (ADP-ribose) polymerase (PARP), key players in apoptosis, were significantly upregulated with the depletion of C-MYC, with or without miR-34a, while Bcl2 expression was significantly reduced. Moreover, proliferating cell nuclear antigen (PCNA) expression levels were significantly suppressed by C-MYC knockdown or miR-34a mimics transfection in SUDHL-6 cells (Fig. 4G). Therefore, significantly altered expression levels of these key markers suggested that the regulation of C-MYC and miR-34a expression levels promoted DCBCL progression via the apoptotic signaling pathway.

Yiqichutan inhibits the growth of DLBCL cells. To investigate the effect of Yiqichutan on DLBCL cells, SUDHL-6 cells were treated with Yiqichutan and viability was measured by MTS (Fig. 5A). It was found that cell viability was inhibited after $4-20 \mathrm{mg} / \mathrm{ml}$ Yiqichutan treatment in a concentration- and time-dependent manner compared with the control group. The $\mathrm{IC}_{50}$ was $14.65 \mathrm{mg} / \mathrm{ml}$ for SUDHL- 6 cells $48 \mathrm{~h}$ after treatment.

Yiqichutan promotes apoptosis of tumor cells. SUDHL-6 cells were treated with different concentrations of Yiqichutan. Flow 

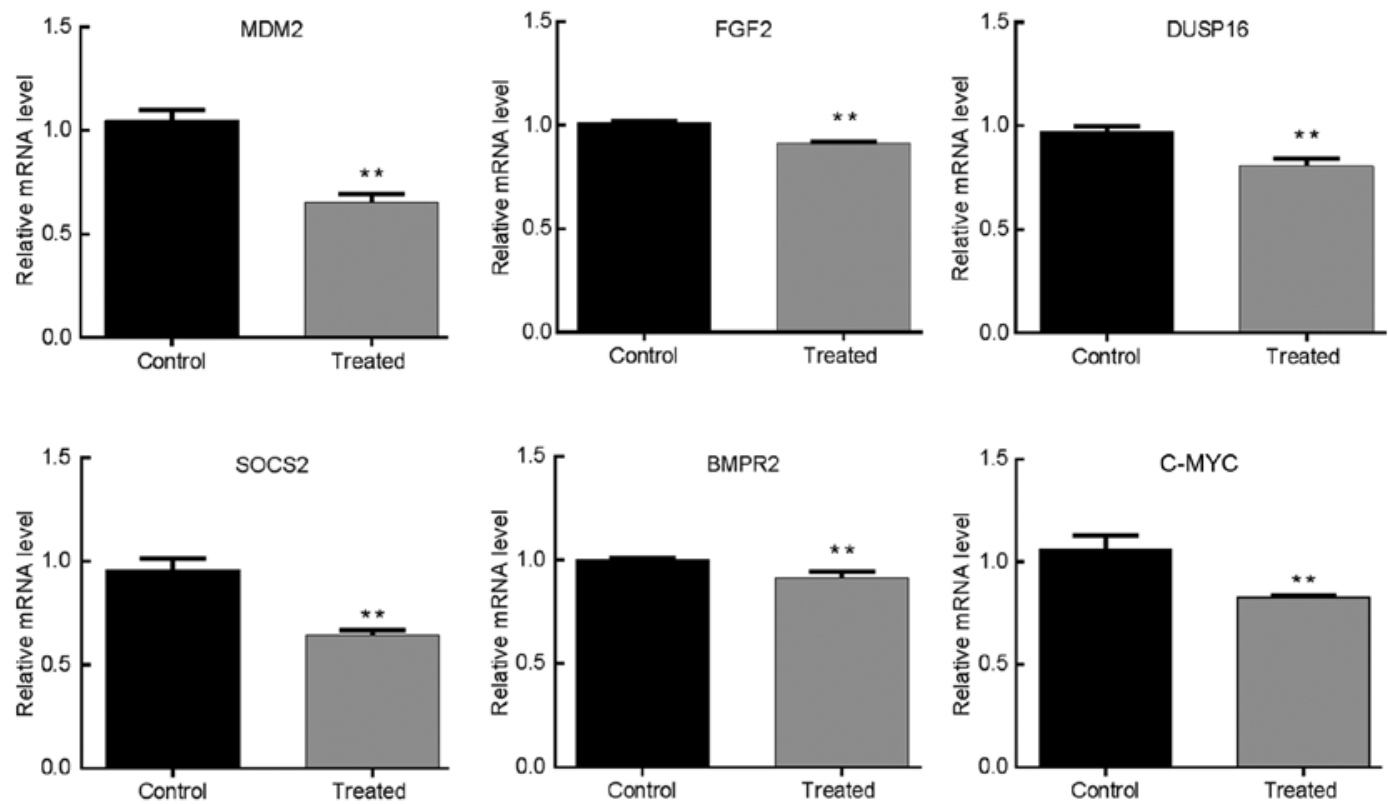

Figure 3. Confirmation of the microarray data by RT-qPCR analysis. In total, six representative mRNAs expressions in DLBCL SUDHL-6 cells were analyzed by RT-qPCR, including C-MYC, MDM2, FGF2, DUSP16, SOCS2 and BMPR2. * P<0.01. RT-qPCR, reverse transcription-quantitative PCR; MDM2, mouse double minute 2; FGF2, fibroblast growth factor 2; DUSP16, Dual specificity protein phosphatase 16; SOCS2, suppressor of cytokine signaling 2; BMPR2, bone morphogenetic protein receptor type 2 .

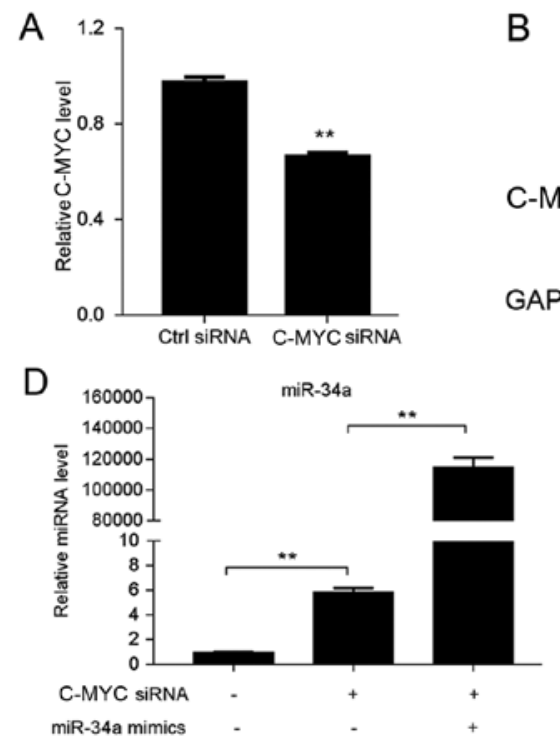

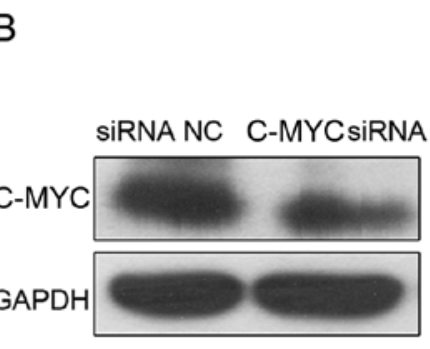

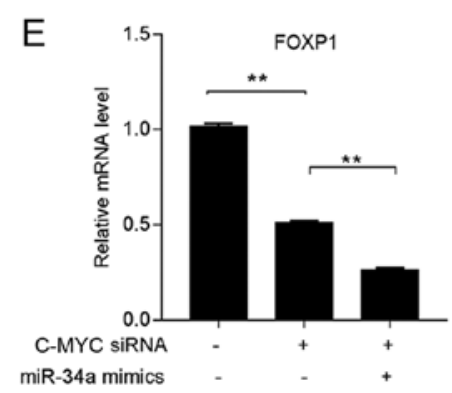

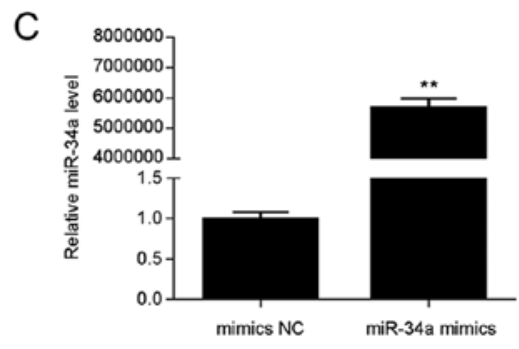

$\mathrm{F}$
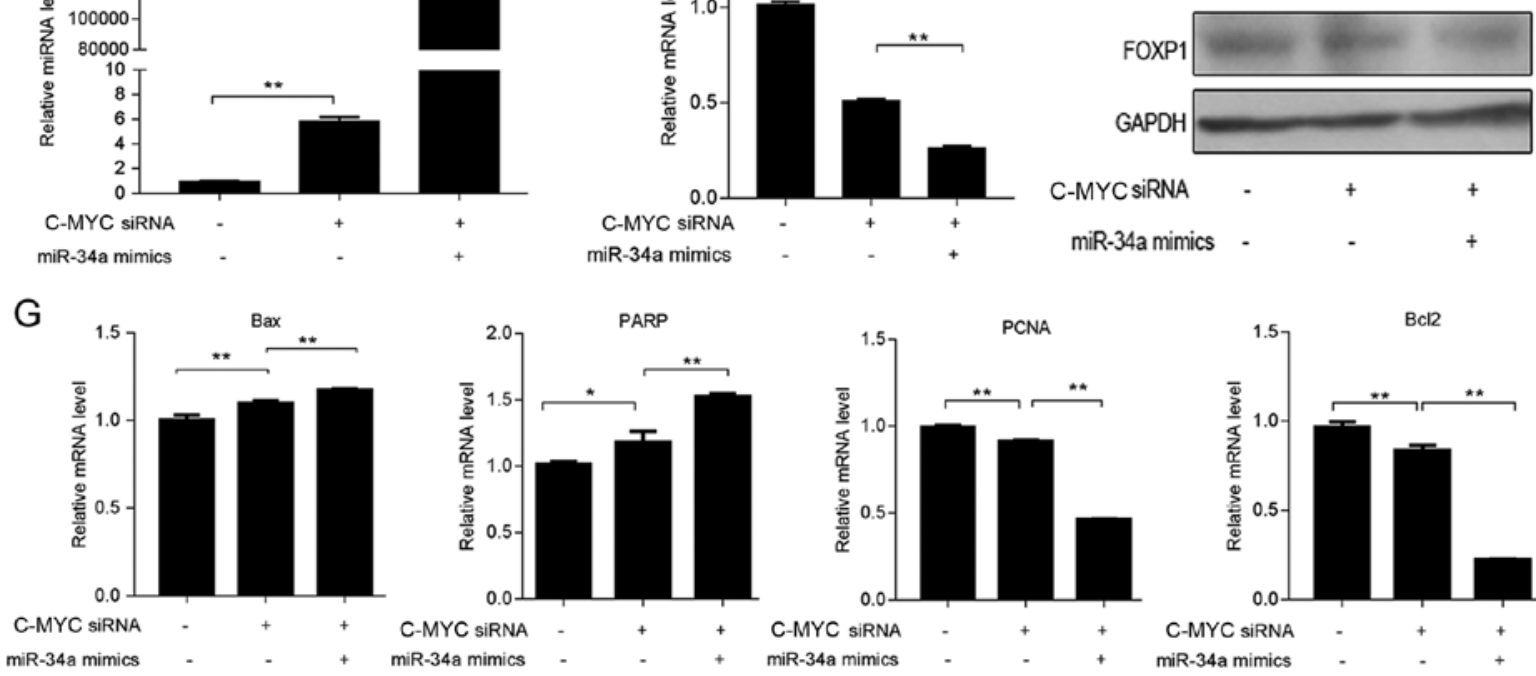

Figure 4. Knockdown of C-MYC increases miR-34a expression level. Expression level of C-MYC in SUDHL-6 cells transfected with siRNAs was determined by (A) RT-qPCR and (B) western blotting. C-MYC siRNA could decrease the expression level of C-MYC. (C) Expression level of miR-34a in SUDHL-6 cells transfected with miR-34a NC and mimics was measured by RT-qPCR. Relative mRNA expression levels of (D) miR-34a and (E) Foxp1 in SUDHL-6 cells transfected with C-MYC siRNAs, with or without miR-34a mimics, were analyzed by RT-qPCR. (F) Protein expression level of Foxp1 was determined by western blotting. Data are presented as the mean \pm SD of three separate experiments. (G) RT-qPCR results indicated that the expression levels of key factors in proliferation and apoptosis, including Bax, Bcl2, PCNA and PARP, were significantly altered with the depletion of C-MYC, with or without miR-34a. "P<0.05, ${ }^{* *} \mathrm{P}<0.01$. RT-qPCR, reverse transcription-quantitative PCR; NC, negative control; miR, microRNA; siRNA, small interfering RNA; Foxp1, forkhead box 1; PCNA, proliferating cell nuclear antigen; PARP, poly (ADP-ribose) polymerase. 

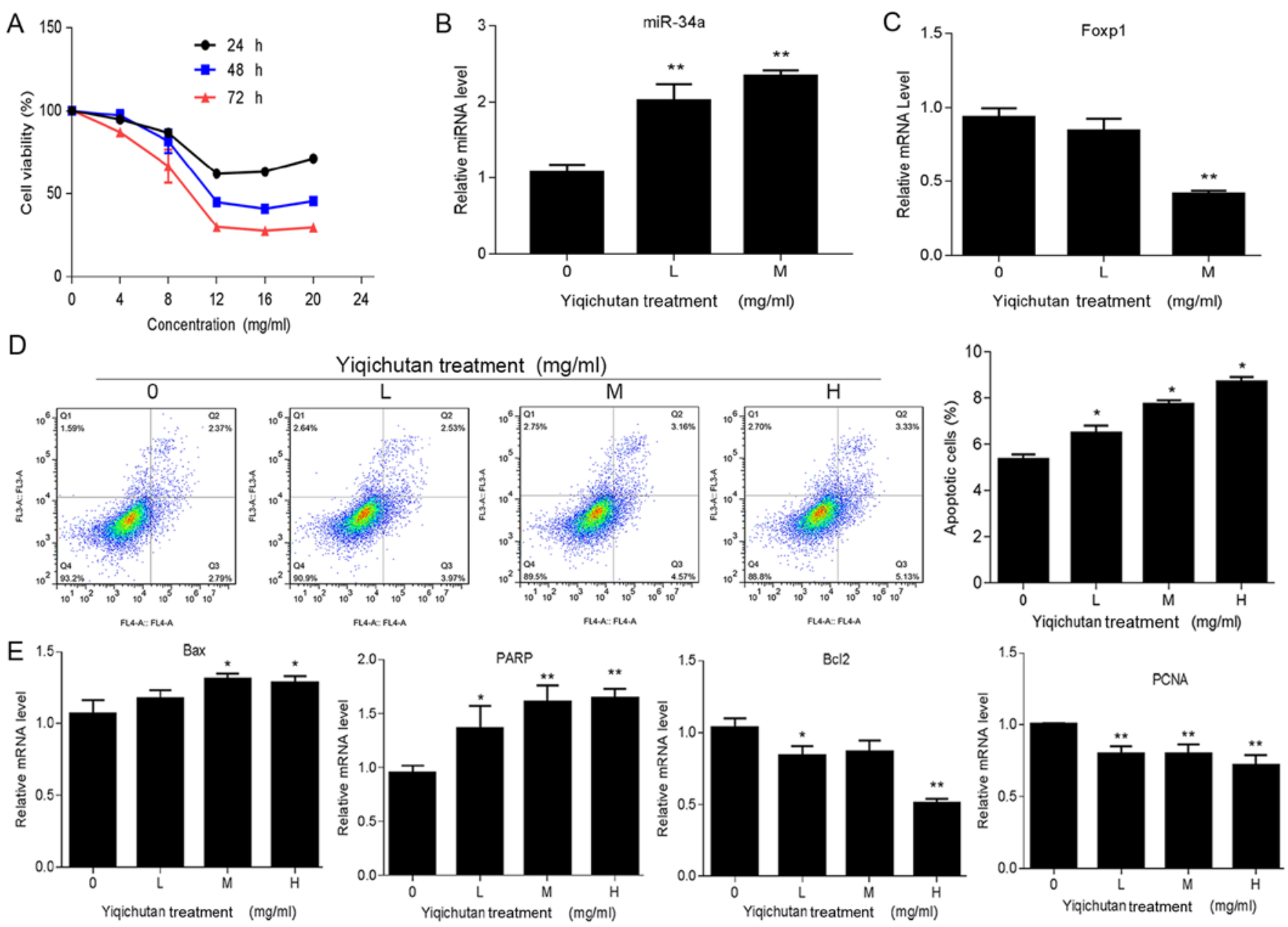

Figure 5. Effect of Yiqichutan on SUDHL-6 cells. (A) SUDHL-6 cells were treated with different concentrations of Yiqichutan. The viability of SUDHL-6 cells was measured with an MTS assay at 24, 48 and $72 \mathrm{~h}$. (B) Cell apoptosis was detected by flow cytometry. (C) Genes associated with proliferation, such as PCNA, and apoptosis, including Bax, Bcl2 and PARP, signaling pathways were measured by RT-qPCR. (D) Expressions levels of miR-34a and Foxp1 in SUDHL-6 cells treated with Yiqichutan were detected by RT-qPCR. (E) RT-qPCR results indicated that the expression levels of Bax, Bcl2, PCNA and PARP, were significantly altered after Yiqichutan treatment. $\mathrm{IC}_{50}=14.65 \mathrm{mg} / \mathrm{ml}$. Data are presented as the mean $\pm \mathrm{SD}$ of three separate experiments. ${ }^{*} \mathrm{P}<0.05$, ${ }^{* *} \mathrm{P}<0.01$. $\mathrm{L}$, low concentration of Yiqichutan $1 / 3 \mathrm{IC}_{50} ; \mathrm{M}$, middle concentration, $1 / 2 \mathrm{IC}_{50} ; \mathrm{H}$, high concentration, $\mathrm{IC}_{50}$. RT-qPCR, reverse transcription-quantitative PCR; miR, microRNA; Foxp1, forkhead box 1; PCNA, proliferating cell nuclear antigen; PARP, poly (ADP-ribose) polymerase.

cytometric analysis results suggested that Yiqichutan treatment promoted cell apoptosis in a concentration-dependent manner (Fig. 5D)

Subsequently, a number of markers associated with cell proliferation and apoptosis were measured by RT-qPCR (Fig. 5E). It was demonstrated that the expression levels of Bax and PARP increased after Yiqichutan treatment, while the Bcl2 expression level was significantly reduced. Furthermore, the PCNA gene, which is associated with cell proliferation (25), was also downregulated in SUDHL-6 cells treated with Yiqichutan. Collectively, the present results indicated that Yiqichutan treatment promoted SUDHL- 6 cell apoptosis.

Yiqichutan promotes miR-34a and reduces Foxpl expression levels in DLBCL cells. To assess whether Yiqichutan could regulate the expression levels of miR-34a and Foxp1, RT-qPCR was performed. It was found that the higher the concentration of Yiqichutan, the higher the expression level of miR-34a (Fig. 5B). However, the expression level of the Foxp1 was reduced, particularly after treatment with the $1 / 2 \mathrm{IC}_{50}$ Yiqichutan (Fig 5C). Therefore, the present results supported the conclusion that Yiqichutan may upregulate miR-34a to promote cell apoptosis and suppress DCBCL progression by reducing the expression level of Foxp1.

\section{Discussion}

The present study use microarrays to investigate possible positive effects of Yiqichutan treatment on DLBCL. The present results suggested that Yiqichutan inhibited DLBCL by acting on several pathways including the Jak/Stat and PI3K signaling pathways, which act as regulators of cell differentiation, migration and proliferation (26). Furthermore, the activation of these pathways can also cause oncogenic transformation and tumor development (26). Several key genes in these pathways such as SOCS2 of the Jak/Stat pathway, MDM2 and FGF2 of the PI3K pathway, and C-MYC of both the Jak/Stat and PI3K pathways are related to oncogenesis and tumor promotion in NHL (27-29). In the present study, it was found that the expression levels of these genes were downregulated after Yiqichutan treatment in vitro, indicating a multi-target antitumor role of Yiqichutan in DLBCL. 
miRNAs are an abundant class of small non-coding RNAs that modulate the expression of their target genes at the post-transcriptional level (30). miR-34a is located on chromosome 1p36.22 in a region associated with various malignancies (31). miR-34a overexpression inhibits the growth of various cancer types in vitro and acts as a tumor suppressor in DLBCL $(12,14,15,18)$. Moreover, high miR-34a expression level improves the host response to doxorubicin in DLBCL (32), and its aberrant expression indicates poor prognosis in gastric MALT lymphoma and DLBCL (33). However, investigating miR-34a function is complicated. Contrary to expectation, the knockdown of endogenous miR-34a results in the inhibition of cell proliferation in chronic lymphocytic leukemia (34). Furthermore, miR-34a overexpression can also show anti-apoptotic effects by compromising the MYC/ADP ribosylation factors/MDM2/p53 axis in MYC-driven lymphomas (35). In the present study, miR-34a was transcriptionally repressed by C-MYC, and knockdown of C-MYC increased the expression level of miR-34a and inhibited cell proliferation in DLBCL. As the upregulation of miR-34a can repress C-MYC, the present results suggested a negative feedback loop between C-MYC and miR-34a, where miR-34a suppresses C-MYC and vice versa. The present results are consistent with those from Craig et al (11) in which epigenetic silencing, both MYC-dependent and -independent, may contribute to miR-34a dysregulation in gastric DLBCL.

Foxp1 functions as an oncogene in controlling tumor development in several malignancies, but its prognostic value in tumors is not fully understood (36). The aberrant expression of Foxp1 is a common feature of DLBCL, indicating a strong negative prognosis indicator of patient survival $(37,38)$. Bioinformatically predicted targets of miR-34a include Foxp1, which harbors putative miR-34a seed regions in its 3 ' untranslated region (21). To assess a possible causal link between miRNA expression and Foxp1 downregulation in DLBCL, the present study knocked down C-MYC and induced miRNA expression in the SUDHL-6 cells. It was found that the transient knockdown decreased the expression level of Foxp1, and knockdown of C-MYC and miR-34a overexpression reduced Foxp1 expression level to a greater extent. Moreover, DLBCL proliferation was significantly reduced after C-MYC knockdown, and the apoptotic rate increased with the change in expression level of the apoptotic factors Bax, Bcl-2 and PARP. In line with results from a previous study, the present results indicated that the C-MYC/miR-34a pathway may have an important role in the apoptotic process of DLBCL (11).

The present study found that Yiqichutan treatment significantly inhibited the growth of SUDHL-6 cells in a concentration- and time-dependent manner. Furthermore, increased apoptosis was identified in treated cells. The mechanism of this inhibition may be related to the miR-34a/Foxp1 pathway, as Yiqichutan increased the expression level of miR-34a and inhibited that of Foxp1. Our previous study showed that Yiqichutan inhibited the growth of DLBCL cells via the regulation of the PI3K/AKT pathway (8). Furthermore, PI3K/AKT inhibition may induce downregulation of the transcription factor C-MYC, as re-expression of C-MYC rescues DLBCL cells from PTEN-induced toxicity (39). Therefore, regulation of the C-MYC/miR-34a pathway by Yiqichutan may be related to its effect on the upstream PI3K/AKT signaling pathway, involving the transcription factor C-MYC.
miR-targeting oligonucleotides have certain advantages as therapeutic tools, most notably the ease with which oligonucleotides can be chemically modified to enhance their pharmacokinetic/pharmacodynamic profiles and the ability of miRNAs to target multiple genes simultaneously (40). A promising strategy to achieve a therapeutic effect by targeting the miRNA regulatory network is to induce the expression of miRNAs that act as tumor suppressor genes (40), such as miR-34a. The first evidence for the success of chemically synthesized miR-34a 'replacement therapy' in cancer treatment was reported using a preclinical model of non-small cell lung cancer (13). In addition, miR-34a administration in a xenotransplant DLBCL and multiple myeloma model resulted in a significant reduction in tumor growth $(41,42)$. The present results indicated the role of Yiqichutan in regulating the C-MYC/miR-34a pathway and increasing the expression levels of miR-34a, which was in accordance with the function of formulated synthetic miR-34a. Thus, the present study identified the potential to develop Yiqichutan-based treatment strategies in patients with DLBCL.

In conclusion, the present study investigated the expression profiles of mRNA in DLBCL cells exposed to Yiqichutan, and found that the C-MYC/miR-34a pathway was associated with the growth of DLBCL cells. Furthermore, Yiqichutan treatment may inhibit the proliferation of DLBCL cells by inhibiting the C-MYC/miR-34a pathway. However, the main limitation of the present study was the lack of in vivo experiments. Thus, further in vivo studies and component analysis of Yiqichutan are required to investigate the mechanism of Yiqichutan treatment in DLBCL cells.

\section{Acknowledgements}

The authors would like to thank Dr Zhuo Lv from people's hospital of Huadu, who provided suggestions for the experimen

\section{Funding}

The current study was supported by grants from the National Natural Science Foundation of China (grant no. 81873147), Guangzhou Municipal Science and Technology Project (grant no. 201607010384), and High-Level University Project of Guangzhou University of Chinese Medicine (grant no. A1-AFD018171Z11069).

\section{Availability of data and materials}

The data that support the results of this study are available from Shanghai GenePharma Co., Ltd., but restrictions apply to the availability of these data, which were used under license for the current study and therefore are not publicly available. Data are, however, available from the authors upon reasonable request and with permission of Shanghai GenePharma Co., Ltd.

\section{Authors' contributions}

LZ designed and performed experiments and wrote the manuscript. YZ performed experiments and wrote the manuscript. JW performed experiments. ZL analyzed the data. 
LL analyzed the data, revised the manuscript and gave final approval of the version to be published. All authors read and approved the final manuscript.

\section{Ethics approval and consent to participate}

Not applicable.

\section{Patient consent for publication}

Not applicable.

\section{Competing interests}

The authors declare that they have no competing interests.

\section{References}

1. Swerdlow SH, Campo E, Pileri SA, Harris NL, Stein H, Siebert R, Advani R, Ghielmini M, Salles GA, Zelenetz AD, et al: The 2016 revision of the World Health Organization classification of lymphoid neoplasms. Blood 127: 2375-2390, 2016.

2. Coiffier B: Rituximab in the treatment of diffuse large B-cell lymphomas. Semin Oncol 29: 30-35, 2002.

3. Habermann TM, Weller EA, Morrison VA, Gascoyne RD, Cassileth PA, Cohn JB, Dakhil SR, Woda B, Fisher RI, Peterson BA, et al: Rituximab-CHOP versus CHOP alone or with maintenance rituximab in older patients with diffuse large B-cell lymphoma. J Clin Oncol 24: 3121-3127, 2006.

4. Sehn LH, Donaldson J, Chhanabhai M, Fitzgerald C, Gill K, Klasa R, MacPherson N, O'Reilly S, Spinelli JJ, Sutherland J, et al: Introduction of combined CHOP plus rituximab therapy dramatically improved outcome of diffuse large B-cell lymphoma in British Columbia. J Clin Oncol 23: 5027-5033, 2005.

5. Park JJ,Beckman-Harned S, Cho G, Kim D and Kim H: The current acceptance, accessibility and recognition of Chinese and Ayurvedic medicine in the United States in the public, governmental, and industrial sectors. Chin J Integr Med 18: 405-408, 2012.

6. Rashrash M, Schommer JC and Brown LM: Prevalence and Predictors of Herbal Medicine Use Among Adults in the United States. J Patient Exp 4: 108-113, 2017.

7. Scheid V, Tuffrey V, Weijburg T, Bovey M and Ward T: Chinese medicine treatment for menopausal symptoms in the UK health service: Is a clinical trial warranted? Maturitas 80: 179-186, 2015.

8. Zhai L, Chen C, Zhao Y and Lin L: Effect of Qi-benefiting and Phlegm-eliminating Recipe on Proliferation and Serine/threonine-protein Kinase AKT Expression of Diffused Large B-cell Lymphoma Cells. J Guangzhou Univ Tradit Chin Med 33: 556-560, 2016 (In Chinese).

9. Gao T, Chen Z and Zhang E: Theoretical Basis and Evidence-based Basis of Invigorating Qi and Expelling Phlegm Method in Preventing and Treating Lung Cancer. Liaoning J Tradit Chin Med 45: 43-46, 2018 (In Chinese).

10. Kim K, Zakharkin SO and Allison DB: Expectations, validity, and reality in gene expression profiling. J Clin Epidemiol 63: 950-959, 2010

11. Craig VJ, Cogliatti SB, Imig J, Renner C, Neuenschwander S, Rehrauer H, Schlapbach R, Dirnhofer S, Tzankov A and Müller A: Myc-mediated repression of microRNA-34a promotes high-grade transformation of B-cell lymphoma by dysregulation of FoxP1. Blood 117: 6227-6236, 2011.

12. Yanaihara N, Caplen N, Bowman E, Seike M, Kumamoto K, Yi M, Stephens RM, Okamoto A, Yokota J, Tanaka T, et al: Unique microRNA molecular profiles in lung cancer diagnosis and prognosis. Cancer Cell 9: 189-198, 2006.

13. Wiggins JF, Ruffino L, Kelnar K, Omotola M, Patrawala L, Brown D and Bader AG: Development of a lung cancer therapeutic based on the tumor suppressor microRNA-34. Cancer Res 70: 5923-5930, 2010.

14. Liu C, Kelnar K, Liu B, Chen X, Calhoun-Davis T, Li H, Patrawala L, Yan H, Jeter C, Honorio S, et al: The microRNA miR-34a inhibits prostate cancer stem cells and metastasis by directly repressing CD44. Nat Med 17: 211-215, 2011.
15. Tang Y, Tang Y and Cheng YS: miR-34a inhibits pancreatic cancer progression through Snaill-mediated epithelial-mesenchymal transition and the Notch signaling pathway. Sci Rep. 7:38232, 2017.

16. Lodygin D, Tarasov V, Epanchintsev A, Berking C, Knyazeva T, Körner H, Knyazev P, Diebold J and Hermeking H: Inactivation of miR-34a by aberrant CpG methylation in multiple types of cancer. Cell Cycle 7: 2591-2600, 2008.

17. Krzeszinski JY, Wei W, Huynh H, Jin Z, Wang X, Chang TC, Xie XJ, He L, Mangala LS, Lopez-Berestein G, et al: miR-34a blocks osteoporosis and bone metastasis by inhibiting osteoclastogenesis and Tgif2. Nature 512: 431-435, 2014.

18. Chim CS, Wong KY, Qi Y, Loong F, Lam WL, Wong LG, Jin DY, Costello JF and Liang R: Epigenetic inactivation of the miR-34a in hematological malignancies. Carcinogenesis 31: 745-750, 2010.

19. Christoffersen NR, Shalgi R, Frankel LB, Leucci E, Lees M, Klausen M, Pilpel Y, Nielsen FC, Oren M and Lund AH: p53-independent upregulation of miR-34a during oncogene-induced senescence represses MYC. Cell Death Differ 17: 236-245, 2010.

20. Jia L, Chopp M, Wang L, Lu X, Zhang Y, Szalad A and Zhang ZG: miR-34a Regulates Axonal Growth of Dorsal Root Ganglia Neurons by Targeting FOXP2 and VAT1 in Postnatal and Adult Mouse. Mol Neurobiol 55: 9089-9099, 2018.

21. Rao DS, O'Connell RM, Chaudhuri AA, Garcia-Flores Y, GeigerTL and Baltimore D: MicroRNA-34a perturbs B lymphocyte development by repressing the forkhead box transcription factor Foxp1. Immunity 33: 48-59, 2010.

22. Kanehisa M, Furumichi M, Tanabe M, Sato Y and Morishima K: KEGG: New perspectives on genomes, pathways, diseases and drugs. Nucleic Acids Res 45 D: D353-D361, 2017.

23. Livak KJ and Schmittgen TD: Analysis of relative gene expression data using real-time quantitative PCR and the 2(-Delta Delta C(T)) Method. Methods 25: 402-408, 2001.

24. Yamada $\mathrm{O}$ and Kawauchi K: The role of the JAK-STAT pathway and related signal cascades in telomerase activation during the development of hematologic malignancies. JAK-STAT 2: e25256, 2013.

25. Dietrich DR: Toxicological and pathological applications of proliferating cell nuclear antigen (PCNA), a novel endogenous marker for cell proliferation. Crit Rev Toxicol 23: 77-109, 1993.

26. Steelman LS, Pohnert SC, Shelton JG, Franklin RA, Bertrand FE and McCubrey JA: JAK/STAT, Raf/MEK/ERK, PI3K/Akt and BCR-ABL in cell cycle progression and leukemogenesis. Leukemia 18: 189-218, 2004.

27. Gupta A, Shah K, Oza MJ and Behl T: Reactivation of p53 gene by MDM2 inhibitors: A novel therapy for cancer treatment. Biomed Pharmacother 109: 484-492, 2019.

28. Song W, Liu MG, Zhang JB, Zhang JJ, Sun MM and Yu QK: Mechanism of action of EBV, Bcl-2, p53, c-Myc and Rb in non-Hodgkin's lymphoma. Eur Rev Med Pharmacol Sci 20: 1093-1097, 2016.

29. Letellier E and Haan S: SOCS2: Physiological and pathological functions. Front Biosci (Elite Ed) 8: 189-204, 2016.

30. Michlewski G and Cáceres JF: Post-transcriptional control of miRNA biogenesis. RNA 25: 1-16, 2019.

31. Calin GA, Sevignani C, Dumitru CD, Hyslop T, Noch E, Yendamuri S, Shimizu M, Rattan S, Bullrich F, Negrini M, et al: Human microRNA genes are frequently located at fragile sites and genomic regions involved in cancers. Proc Natl Acad Sci USA 101: 2999-3004, 2004.

32. Marques SC, Ranjbar B, Laursen MB, Falgreen S, Bilgrau AE, Bødker JS, Jørgensen LK, Primo MN, Schmitz A, Ettrup MS, et al: High miR-34a expression improves response to doxorubicin in diffuse large B-cell lymphoma. Exp Hematol 44: 238-246 e232, 2016.

33. He M, Gao L, Zhang S, Tao L, Wang J, Yang J and Zhu M: Prognostic significance of miR-34a and its target proteins of FOXP1, p53, and BCL2 in gastric MALT lymphoma and DLBCL. Gastric Cancer 17: 431-441, 2014.

34. Saleh LM, Wang W, Herman SE, Saba NS, Anastas V, Barber E, Corrigan-Cummins M, Farooqui M, Sun C, Sarasua SM, et al: Ibrutinib downregulates a subset of miRNA leading to upregulation of tumor suppressors and inhibition of cell proliferation in chronic lymphocytic leukemia. Leukemia 31: 340-349, 2017.

35. Musilova K and Mraz M: MicroRNAs in B-cell lymphomas: How a complex biology gets more complex. Leukemia 29: 1004-1017, 2015. 
36. Xiao J, He B, Zou Y, Chen X, Lu X, Xie M, Li W, He S, You S and Chen Q: Prognostic value of decreased FOXP1 protein expression in various tumors: A systematic review and meta-analysis. Sci Rep 6: 30437, 2016.

37. Flori M, Schmid CA, Sumrall ET, Tzankov A, Law CW Robinson MD and Müller A: The hematopoietic oncoprotein FOXP1 promotes tumor cell survival in diffuse large B-cell lymphoma by repressing S1PR2 signaling. Blood 127: 1438-1448, 2016.

38. Gascoyne DM and Banham AH: The significance of FOXP1 in diffuse large B-cell lymphoma. Leuk Lymphoma 58: 1037-1051,2017.

39. Pfeifer M, Grau M, Lenze D, Wenzel SS, Wolf A, Wollert-Wulf B, Dietze K, Nogai H, Storek B, Madle H, et al: PTEN loss defines a PI3K/AKT pathway-dependent germinal center subtype of diffuse large B-cell lymphoma. Proc Natl Acad Sci USA 110: 12420-12425, 2013.

40. Li Z and Rana TM: Therapeutic targeting of microRNAs: Current status and future challenges. Nat Rev Drug Discov 13: $622-638,2014$
41. Craig VJ, Tzankov A, Flori M, Schmid CA, Bader AG and Müller A: Systemic microRNA-34a delivery induces apoptosis and abrogates growth of diffuse large B-cell lymphoma in vivo. Leukemia 26: 2421-2424, 2012.

42. Di Martino MT, Leone E, Amodio N, Foresta U, Lionetti M, Pitari MR, Cantafio ME, Gullà A, Conforti F, Morelli E, et al: Synthetic miR-34a mimics as a novel therapeutic agent for multiple myeloma: in vitro and in vivo evidence. Clin Cancer Res 18: 6260-6270, 2012.

This work is licensed under a Creative Commons Attribution-NonCommercial-NoDerivatives 4.0 International (CC BY-NC-ND 4.0) License. 